\title{
Parametric earthquake analysis of thick plates using Mindlin's theory
}

\author{
Y.I. Özdemir and Y. Ayvaz* \\ Department of Civil Engineering, Karadeniz Technical University, 61080 Trabzon, Turkey
}

Received 14 February 2008

Revised 29 April 2009

\begin{abstract}
The purpose of this paper is to study parametric earthquake analysis of thick plates using Mindlin's theory, to determine the effects of the thickness/span ratio, the aspect ratio and the boundary conditions on the linear responses of thick plates subjected to earthquake excitations and to present the frequency parameters and the mode shapes of the same plates. In the analysis, finite element method is used for spatial integration and the Newmark- $\beta$ method is used for time integration. A computer program using finite element method is coded in $\mathrm{C}++$ to analyze the plates clamped or simply supported along all four edges. In the analysis, 8-noded finite element is used. Graphs and tables are presented that should help engineers in the design of thick plates subjected to earthquake excitations. It is concluded that, in general, the changes in the thickness/span ratio are more effective on the maximum responses considered in this study than the changes in the aspect ratio. It is also concluded that the effects of the change in the thickness/span ratio on the frequency parameter of the thick plates are always larger than those of the change in the aspect ratio.
\end{abstract}

Keywords: Parametric earthquake analysis, thick plate, Mindlin's theory, finite element method, thickness/span ratio, aspect ratio, boundary conditions, free vibration analysis

\section{Introduction}

Plates are structural elements which are commonly used in the building industry. A plate is considered to be a thin plate if the ratio of the plate thickness to the smaller span length is less than $1 / 20$; it is considered to be a thick plate if this ratio is larger than $1 / 20[1,32]$.

The dynamic behavior of thin plates has been investigated by many researchers $[3,4,6,7,13,14,16,17,30,33,36,38$, 39]. There are also many references on the behavior of the thick plates subjected to different loads. The studies made on the behavior of the thick plates are based on the Reissner-Mindlin plate theory [9-11,29]. This theory requires only $\mathrm{C}^{0}$ continuity for the finite elements in the analysis of thin and thick plates. Therefore, it appears as an alternative to the thin plate theory which also requires $\mathrm{C}^{1}$ continuity. This requirement in the thin plate theory is solved easily if Mindlin theory is used in the analysis of thin plates. Despite the simple formulation of this theory, discretization of the plate by means of the finite element comes out to be an important parameter. In many cases, numerical solution can have lack of convergence, which is known as "shear-locking". Shear locking can be avoided by increasing the mesh size, i.e. using finer mesh, but if the thickness/span ratio is "too small", convergence may not be achieved even if the finer mesh is used for the low order displacement shape functions.

In order to avoid shear locking problem, the different methods and techniques, such as reduced and selective reduced integration, the substitute shear strain method, etc., are used by several researchers $[8,26,27,34,35]$. The same problem can also be prevented by using higher order displacement shape function [41]. Wanji and Cheung [5]

\footnotetext{
*Corresponding author. Tel.: +90 462 3773238; Fax: +90 462 3772606; E-mail: ayvaz@ktu.edu.tr.
} 


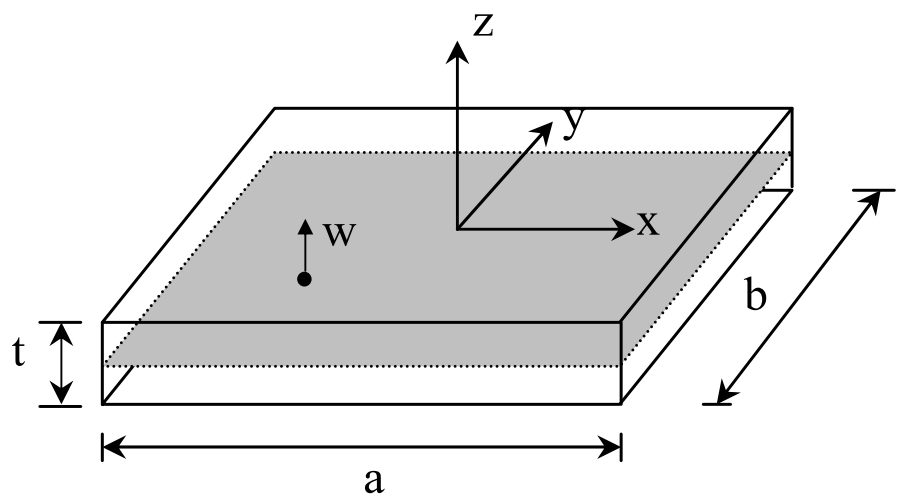

Fig. 1. The sample plate used in this study.

proposed a new quadrilateral thin/thick plate element based on the Mindlin-Reissner theory. Soh et al. [2] improve a new element ARS-Q12 which is a simple quadrilateral 12 DOF plate bending element based on Reissner-Mindlin theory for analysis of thick and thin plates.. Brezzi and Marini [12] developped a locking free nonconforming element for the Reissner-Mindlin plate using discontinuous Galarkin techniques. Belounar and Guenfound [22] improved a nev rectangular finite element based on the strain approach and the Reissner-Mindlin theory is presented for the analysis of plates in bending either thick or thin. Cen et al. [31] developed a new high performance quadrilateral element for analysis of thick and thin plates. This distinguishing character of the new element is that all formulations are expressed in the quadrilateral area co-ordinate system. Ayvaz [39] derived the equations of motions for thick orthotropic elastic plates using Hamilton's principle, but did not present any results. Liew and Teo [20] studied three-dimensional vibration analysis of rectangular plates based on differential quadrature method. Shen et al. [15] studied free and forced vibration of Reissner-Mindlin plates with free edges resting on elastic foundations. Cai et al. [23] presented the generalized mixed variational principle for Reissner plate analysis. Using finite element method, Raju and Hinton [19] made significant contributions to the vibration analysis of plates including rotatory inertia effects for rhombic plates based on Mindlin's theory. Woo et al. [21] found accurate natural frequencies and mode shapes of skew plates with and without cutouts by p-version finite element method using integrals of Legendre polynomial for $p=1-14$. Qian et al. [24] studied free and forced vibrations of thick rectangular plates using higher-order shear and normal deformable plate theory and meshless Petrov-Galarkin method. Morais et al. [25] studied vibrations of thick plates using Lagrangean quadrilateral finite element with 16 nodes. However, no references have been found in the technical literature for the earthquake analysis of thick plates using Mindlin theory.

The purpose of this paper is to study parametric earthquake analysis of thick plates using Mindlin's theory, to determine the effects of the thickness/span ratio, the aspect ratio and the boundary conditions on the linear responses of the thick plates subjected to earthquake excitations and to present the frequency parameters and the mode shapes of the same plates. A computer program using finite element method is coded in $\mathrm{C}++$ to analyze the plates clamped or simply supported along all four edges. In the program, the finite element method is used for spatial integration and the Newmark- $\beta$ method is used for the time integration. In the analysis, 8 -noded finite elements are used to construct the stiffness and mass matrices since shear locking problem does not occur if this element is used in the finite element modeling of the thick plates [41,42].

\section{Mindlin's theory of thick plates}

In the plate of Fig. 1, assuming that $u$ and $v$ are proportional to $z$ and $w$ is independent of $z$, the plate displacement in terms of the two slopes and a displacement at an arbitrary $x, y$, and $z$ point can be written as follows:

$$
u=\{u, v, w\}=\left\{-z \phi_{x}, z \phi_{y}, w\right\}=\left\{-z \frac{\partial \varphi_{i}}{\partial x}, z \frac{\partial \varphi_{i}}{\partial y}, w\right\}
$$



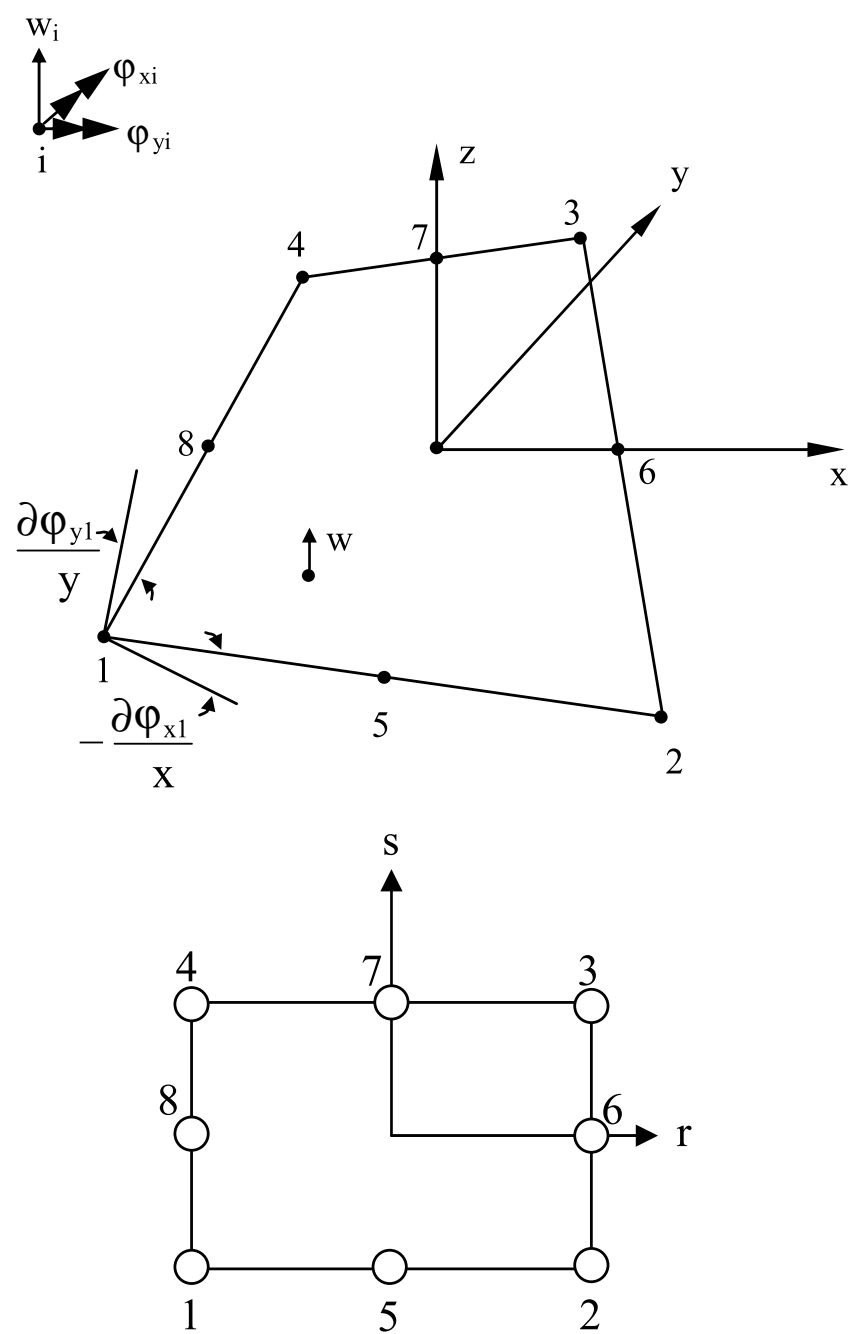

Fig. 2. 8-noded quadrilateral finite element used in this study [41].

In this equation, $u, v, w$ are displacements in the $x, y$, and $z$ directions, respectively, $x, y$, and $z$ are the co-ordinate axes and $\varphi_{x}$ and $\varphi_{y}$ are the rotations in the $x$ and $y$ directions, respectively (Fig. 2). The equations for strain components in terms of plate displacementsu, $v$, and $w$ are given as follows [39]:

$$
\begin{aligned}
\varepsilon_{x}=\frac{\partial u}{\partial x} & \gamma_{x y}=\frac{\partial v}{\partial x}+\frac{\partial u}{\partial y} \\
\varepsilon_{y}=\frac{\partial v}{\partial y} & \gamma_{x z}=\frac{\partial w}{\partial x}+\frac{\partial u}{\partial z} \\
\varepsilon_{z}=\frac{\partial w}{\partial z} & \gamma_{y z}=\frac{\partial w}{\partial y}+\frac{\partial v}{\partial z}
\end{aligned}
$$

The expressions for stresses are given in terms of stress resultants as follows [10]:

$$
\begin{array}{ll}
\sigma_{x}=\frac{12 M_{x} z}{t^{3}} & \tau_{x y}=\frac{12 M_{x y} z}{t^{3}} \\
\sigma_{y}=\frac{12 M_{y} z}{t^{3}} & \tau_{x z}=\frac{3 Q_{x} z}{2 t}\left(1-\frac{4 z^{2}}{t^{2}}\right) \\
\sigma_{z}=0 & \tau_{x z}=\frac{3 Q_{y} z}{2 t}\left(1-\frac{4 z^{2}}{t^{2}}\right)
\end{array}
$$


where is the plate thickness, $M_{x}$, and $M_{y}$ are bending moments, $M_{x y}$ is twisting moment, $Q_{x}$, and $Q_{y}$ are shear forces. In order to derive equations of motion for a plate, Hamilton's equation is used. This equation is given as follows:

$$
\delta \int_{t_{1}}^{t_{2}}(T-V) d t=0
$$

where $\mathrm{T}$ is the kinetic energy of the plate, and $V$ is the potential energy of the plate. The kinetic energy of the plate is given as follows:

$$
T=\frac{1}{2} \iiint m\left(\ddot{u}^{2}+\ddot{v}^{2}+\ddot{w}^{2}\right) d v
$$

where $\mathrm{m}$ is mass per unit volume. The potential energy function, $V$, for a plate subjected to an earthquake excitation is given as follows:

$$
V=F-\iint \bar{q} w d x d y
$$

where $\bar{q}$ denotes $-m \ddot{u}_{g}, \ddot{u}_{g}$ is the earthquake acceleration, and $F$, the strain energy, is written as follows [10]:

$$
F=\int_{v}\left(\sigma_{x} \varepsilon_{x}+\sigma_{y} \varepsilon_{y}+\sigma_{z} \varepsilon_{z}+\tau_{x y} \gamma_{x y}+\tau_{x z} \gamma_{x z}+\tau_{y z} \gamma_{y z}\right) d v-\bar{W}
$$

and $\bar{W}$ is the complementary energy of the plate, which is given as follows:

$$
\bar{W}=\frac{1}{2} \int_{v}\left(\sigma_{x} \varepsilon_{x}+\sigma_{y} \varepsilon_{y}+\sigma_{z} \varepsilon_{z}+\tau_{x y} \gamma_{x y}+\tau_{x z} \gamma_{x z}+\tau_{y z} \gamma_{y z}\right) d v
$$

By substituting Eqs (2) and (3) into Eqs (7) and (8), Equation (8) into Eq. (7), Eq. (7) into Eq. (6), Eqs (5) and (6) into Eq. (4), the following dynamic and stress resultant equations from Hamilton's principle can be obtained;

Dynamic equations:

$$
\begin{aligned}
& \frac{\partial M_{x}}{\partial x}+\frac{\partial M_{x y}}{\partial y}-Q_{x}=\frac{m t^{3}}{12} \ddot{\varphi}_{x} \\
& \frac{\partial M_{x y}}{\partial x}+\frac{\partial M_{y}}{\partial y}-Q_{y}=\frac{m t^{3}}{12} \ddot{\varphi}_{y} \\
& \frac{\partial Q_{x}}{\partial x}+\frac{\partial Q_{y}}{\partial y}-m \ddot{u}_{g}=m h \ddot{w}
\end{aligned}
$$

Equation for stress resultants:

$$
\begin{aligned}
M_{x} & =\bar{D}\left(-\frac{\partial \varphi_{x}}{\partial x}+\nu \frac{\partial \varphi_{y}}{\partial y}\right) \\
M_{y} & =\bar{D}\left(\frac{\partial \varphi_{y}}{\partial y}-\nu \frac{\partial \varphi_{x}}{\partial x}\right) \\
M_{x y} & =D_{t}\left(-\frac{\partial \varphi_{x}}{\partial y}+\frac{\partial \varphi_{y}}{\partial x}\right) \\
Q_{x} & =C\left(-\varphi_{x}+\frac{\partial w}{\partial x}\right) \\
Q_{y} & =C\left(\varphi_{y}+\frac{\partial w}{\partial y}\right)
\end{aligned}
$$

The equations to calculate $C, \bar{D}, D_{t}$ are given as follows: 


$$
\begin{aligned}
C & =\frac{5}{6} G t \\
\bar{D} & =\frac{E t^{3}}{12\left(1-\nu^{2}\right)} \\
D_{t} & =\frac{G t^{3}}{12}
\end{aligned}
$$

where $E, G, \nu$, and $t$ are modulus of the elasticity, shear modulus, Poisson's ratio, and the thickness of the plate, respectively,

By substituting Eq. (10) into Eq. (9), the equation of motion for a plate subjected to earthquake excitations can be obtained. These equations are as follows:

$$
\begin{aligned}
& \bar{D}\left(-\frac{\partial^{2} \varphi_{x}}{\partial x^{2}}+\nu \frac{\partial^{2} \varphi_{y}}{\partial x \partial y}\right)+D_{t}\left(-\frac{\partial^{2} \varphi_{x}}{\partial y^{2}}+\frac{\partial^{2} \varphi_{y}}{\partial x \partial y}\right)-c\left(-\varphi_{x}+\frac{\partial w}{\partial x}\right)=\frac{m h^{3}}{12} \frac{\partial^{2} \varphi_{x}}{\partial t^{2}} \\
& \bar{D}\left(\frac{\partial^{2} \varphi_{y}}{\partial y^{2}}-\nu \frac{\partial^{2} \varphi_{x}}{\partial x \partial y}\right)+D_{t}\left(-\frac{\partial^{2} \varphi_{x}}{\partial x \partial y}+\frac{\partial^{2} \varphi_{y}}{\partial y^{2}}\right)-c\left(\varphi_{y}+\frac{\partial w}{\partial y}\right)=\frac{m h^{3}}{12} \frac{\partial^{2} \varphi_{y}}{\partial t^{2}} \\
& c\left(-\frac{\partial \varphi_{x}}{\partial x}+\frac{\partial^{2} w}{\partial x^{2}}\right)+c\left(\frac{\partial \varphi_{y}}{\partial y}+\frac{\partial^{2} w}{\partial y^{2}}\right)-m \ddot{u}_{g}=m h \frac{\partial^{2} w}{\partial t^{2}}
\end{aligned}
$$

In the case of the free vibration analysis, the term, $-m \ddot{u}_{g}$, in the third equation of Eq. (12) becomes zero. In this study, finite element method is used to solve the plate governed by Eq. (12).

\section{Finite element modeling}

The governing equation for a flexural plate (see Fig. 1) subjected to free vibration without damping can be given, in matrix notation, as

$$
[M]\{\ddot{w}\}+[K]\{w\}=0
$$

where $[K]$ and $[M]$ are the stiffness matrix and the mass matrix of the plate, respectively, $w$ and $\ddot{w}$ are the lateral displacement and the second derivative of the lateral displacement of the plate with respect to time, respectively.,

The governing equation for a flexural plate subjected to an earthquake excitation without damping can be given, in matrix notation, as

$$
[M]\{\ddot{w}\}+[K]\{w\}=[F]=-[M]\left\{\ddot{u}_{g}\right\}
$$

In order to do free and forced vibration analysis of a plate, the stiffness, $[K]$, mass matrices, $[M]$, and equivalent nodal loads vector, $[F]$, of the plate should be constructed. The evaluation of these matrices is given in the following sections.

\subsection{Evaluation of the stiffness matrix}

The simplest type of plate-bending element has rectangular geometry which is a special case of quadrilateral geometry. In this study, 8-noded quadrilateral serendipity element (MT8) (see Fig. 2) is used. The stiffness matrix for this element can be obtained by the following equation [28].

$$
[K]=\int_{A}[B]^{T}[D][B] d A=\int_{-1}^{1} \int_{-1}^{1}[B]^{T}[D][B]|J| \operatorname{drds}
$$

which must be evaluated numerically [24].

As seen from Eq. (15), in order to obtain the stiffness matrix, $[B]$, and the flexural rigidity matrix, $[D]$, of the element need to be constructed.

The nodal displacements for 8-noded finite element can be written as follows; 


$$
u=-z \phi_{x}=-z \sum_{i=1}^{8} h_{i} \varphi_{x i}, v=z \phi_{y}=z \sum_{i=1}^{8} h_{i} \varphi_{y i}, w=\sum_{i=1}^{8} h_{i} w_{i}
$$

The displacement function chosen for this element is;

$$
w=c_{1}+c_{2} r+c_{3} s+c_{4} r^{2}+c_{5} r s+c_{6} s^{2}+c_{7} r^{2} s+c_{8} r s^{2}
$$

where $r$ and $s$ are the local coordinates of the plate.

From this assumption, it is possible to derive the displacement shape function $h$ to be;

$$
h=\left[h_{1}, h_{2}, h_{3}, h_{4}, h_{5}, h_{6}, h_{7}, h_{8}\right]
$$

where [41]

$$
\begin{aligned}
& h_{1}=\left(\frac{1}{4}\right) *(1-r) *(1-s) *(-r-s-1), \quad h_{2}=\left(\frac{1}{2}\right) *(1-r * r) *(1-s) \\
& h_{3}=\left(\frac{1}{4}\right) *(1+r) *(1-s) *(r-s-1), \quad h_{4}=\left(\frac{1}{2}\right) *(1+r) *(1-s * s) \\
& h_{5}=\left(\frac{1}{4}\right) *(1+r) *(1+s) *(r+s-1), \quad h_{6}=\left(\frac{1}{2}\right) *(1-r * r) *(1+s) \\
& h_{7}=\left(\frac{1}{4}\right) *(1-r) *(1+s) *(-r+s-1), \quad h_{8}=\left(\frac{1}{2}\right) *(1-r) *(1-s * s) .
\end{aligned}
$$

Then, the strain-displacement matrix $[B]$ for this element can be written as follows [37]:

$$
[B]=\left[\begin{array}{cccc}
0 & 0 & -\frac{\partial h_{i}}{\partial x} & \cdots \\
0 & \frac{\partial h_{i}}{\partial y} & 0 & \cdots \\
0 & \frac{\partial h_{i}}{\partial x} & -\frac{\partial h_{i}}{\partial y} & \cdots \\
\frac{\partial h_{i}}{\partial x} & 0 & -h_{i} & \cdots \\
\frac{\partial h_{i}}{\partial y} & h_{i} & 0 & \cdots
\end{array}\right]_{5 x 21}
$$

The flexural rigidity matrix, $[D]$, can be obtained by the following equation.

$$
[D]=\left[\begin{array}{cc}
E_{k} & 0 \\
0 & E_{\gamma}
\end{array}\right] \text {. }
$$

In this equation, $\left[E_{k}\right]$ is of size $3 \times 3$ and $\left[E_{\gamma}\right]$ is of size $2 \times 2 .\left[E_{k}\right]$, and $\left[E_{\gamma}\right]$ can be written as follows [18]:

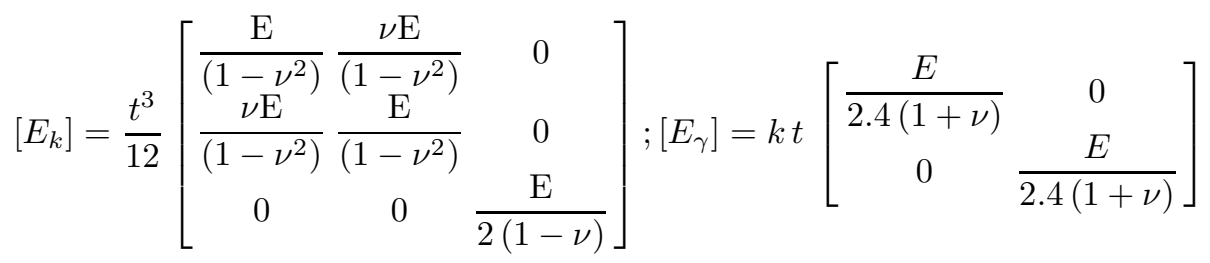

where $k$ is a constant to account for the actual non-uniformity of the shearing stresses. By assembling the element stiffness matrices obtained, the system stiffness matrix is obtained.

\subsection{Evaluation of the mass matrix}

The formula for the consistent mass matrix of the plate may be written as

$$
M=\int_{\Omega} H_{i}^{T} \mu H_{i} d \Omega .
$$

In this equation, $\mu$ is the mass density matrix of the form [40]

$$
\mu=\left[\begin{array}{ccc}
m_{1} & 0 & 0 \\
0 & m_{2} & 0 \\
0 & 0 & m_{3}
\end{array}\right],
$$


where $\mathrm{m}_{1}=\rho_{p} \mathrm{t}, \mathrm{m}_{2}=\mathrm{m}_{3}=\frac{1}{12}\left(\rho_{p} t^{3}\right)$, and $\rho_{p}$ is the mass densities of the plate. and $H_{i}$ can be written as follows,

$$
H_{i}=\left[\begin{array}{lll}
d h_{i} / d x & d h_{i} / d y & h_{i}
\end{array}\right] \quad i=1 \quad \ldots \quad 8 .
$$

It should be noted that the rotation inertia terms are not taken into account. By assembling the element mass matrices obtained, the system mass matrix is obtained.

\subsection{Evaluation of equivalent nodal loads vector}

Equivalent nodal loads, $[F]$, can be obtained by the following equation.

$$
[F]=\int H_{i}^{T} \bar{q} d \Omega
$$

In this equation, $\mathrm{H}_{i}$ can be obtained by Eq. (25).

It should be noted that, in this study, the program, MATLAB, is used for the eigenvalue solution of Eq. (13). It should also be noted that, the Newmark- $\beta$ method is used for the time integration of Eq. (14) by using the average acceleration method.

\section{Numerical examples}

\subsection{Data for numerical examples}

In the light of the results given in references [41,42], the aspect ratios, b/a, of the plate are taken to be $1,1.5$, and 2.0. The thickness/span ratios, $t / a$ are taken as $0.05,0.1,0.2$, and 0.3 for each aspect ratio. The shorter span length of the plate is kept constant to be $3 \mathrm{~m}$. The mass density, Poisson's ratio, and the modulus of elasticity of the plate are taken to be $2.5 \mathrm{kN} \mathrm{s}^{2} / \mathrm{m}^{2}, 0.2$, and $2.8 \times 10^{7} \mathrm{kN} / \mathrm{m}^{2}$ for both analysis. In order to obtain the response of each plate in the forced vibration analysis by using the time history analysis, the East-West component of March 131992 Erzincan earthquake in Turkey is used. Duration of this earthquake is $21 \mathrm{~s}$, but, in this study, the first $8 \mathrm{~s}$ of the earthquake is used since the peak value of the record occurred in this range (Fig. 3 (a)). The acceleration spectrum curve of the same component of this earthquake is also given in Fig. 3(b).

For the sake of accuracy in the results, rather than starting with a set of a finite element mesh size and time increment, the mesh size and time increment required to obtain the desired accuracy were determined before presenting any results. This analysis was performed for the mesh size in the free vibration analysis. This analysis was also performed separately for the mesh size and time increment in the forced vibration analysis. It was concluded that the results have acceptable error when equally spaced $16 \times 16$ mesh sizes are used for a $3 \mathrm{~m} \times 3 \mathrm{~m}$ plate for the free vibration analysis. It was also concluded that the results have acceptable error when equally spaced $16 \times 16$ mesh sizes are used for a $3 \mathrm{~m} \times 3 \mathrm{~m}$ plate for the forced vibration analysis, if the $0.01 \mathrm{~s}$ time increment is used. Length of the elements in the $x$ and $y$ directions are kept constant for different aspect ratios as in the case of square plate.

\subsection{Results}

\subsubsection{Free vibration results}

The first six frequency parameters of thick plates considered for different aspect ratio, $b / a$, thickness/smaller span ratio, $t / a$, are presented in Table 1 for the thick simply supported plates and in Table 2 for thick clamped plates. In order to see the effects of the changes in these parameters better on the first six frequency parameters, they are also presented in Fig. 4 for the thick simply supported plates and in Fig. 5 for the thick clamped plates.

As seen from Tables 1 and 2, and Figs 4 and 5, the values of the frequency parameters for a constant value of $t / a$ decrease as the aspect ratio, $b / a$, increases. This behavior is understandable in that a thick plate with a larger aspect ratio becomes more flexible and has smaller frequency parameters. The decreases in the frequency parameters with increasing value of $\mathrm{b} / \mathrm{a}$ ratio gets less for a constant value of $t / a$. 


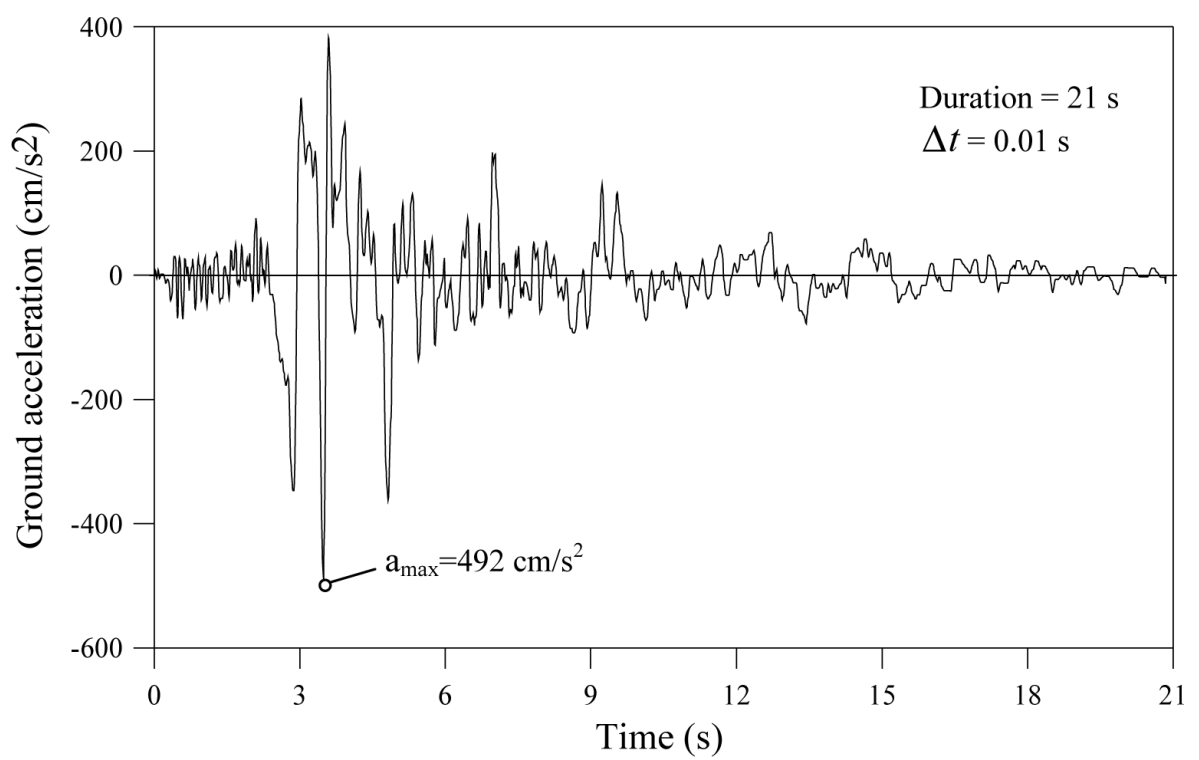

(a)

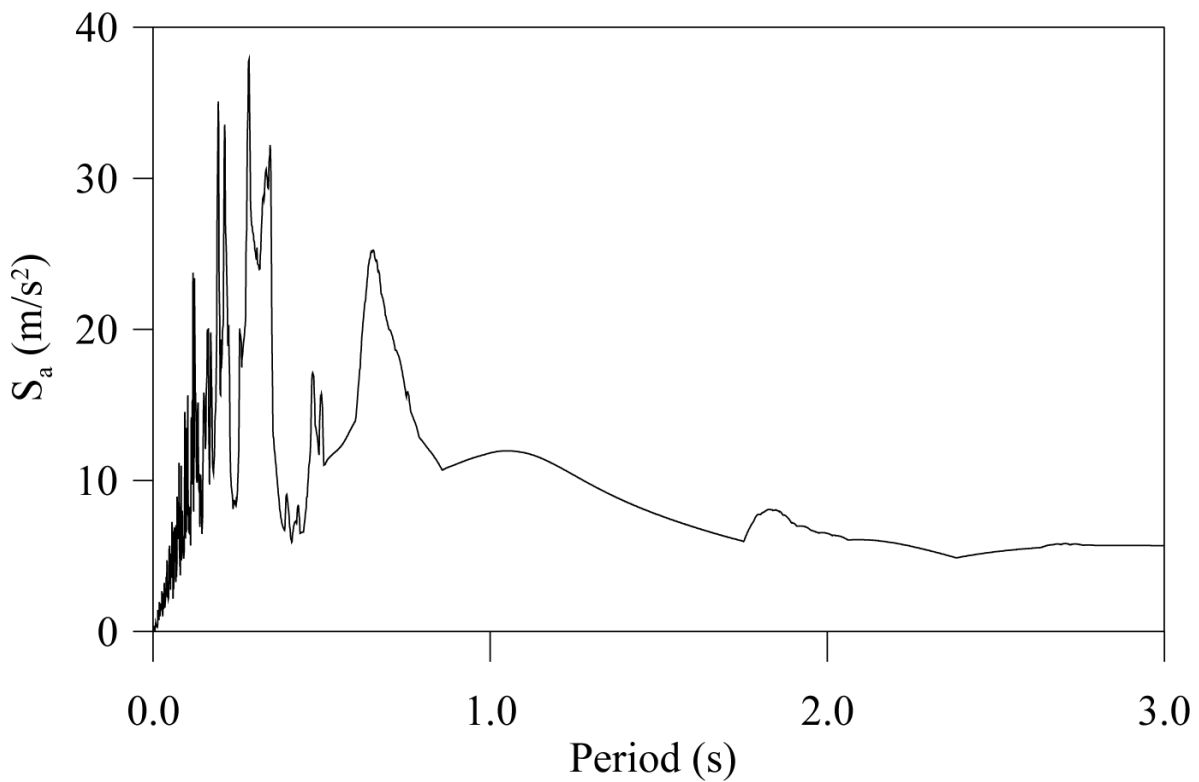

(b)

Fig. 3. (a) East-West component of the March 13, 1992 Erzincan earthquake in Turkey and (b) its acceleration spectrum.

As also seen from Tables 1 and 2, and Figs 4 and 5, the values of the frequency parameters for a constant value of $b / a$ increase as the thickness/span ratio, $b / a$, increases. This behavior is also understandable in that a thick plate with a larger thickness/span ratio becomes more rigid and has larger frequency parameters. The increases in the frequency parameters with increasing value of $t / a$ ratio gets larger for a constant value of $b / a$.

It should be noted that the increase in the frequency parameters with increasing $t / a$ ratios for a constant value of $b / a$ ratio gets larger for larger values of the frequency parameters. These observations indicate that the effects of the change in the $t / a$ ratio on the frequency parameter of the thick plates simply supported or clamped along all four edges are always larger than those of the change in the aspect ratio.

As also seen from Figs 4 and 5, the curves for a constant value of the aspect ratio, $b / a$ are fairly getting closer to 
Table 1

The first six natural frequency parameters of thick simply supported plates for different $b / a$ ve $t / a$ ratios

\begin{tabular}{clcccccc}
\hline \multirow{2}{*}{$\mathrm{b} / \mathrm{a}$} & $\mathrm{t} / \mathrm{a}$ & \multicolumn{7}{c}{$\lambda_{i}=\omega \mathrm{t} \sqrt{\frac{\rho}{E}}$} \\
\cline { 3 - 8 } & & $\lambda_{1}$ & $\lambda_{2}$ & $\lambda_{3}$ & $\lambda_{4}$ & $\lambda_{5}$ & $\lambda_{6}$ \\
\hline \multirow{4}{*}{1.0} & 0.05 & 0.0141 & 0.0354 & 0.0354 & 0.0555 & 0.0698 & 0.0698 \\
& 0.1 & 0.0545 & 0.1321 & 0.1321 & 0.2004 & 0.2496 & 0.2501 \\
& 0.2 & 0.1945 & 0.4389 & 0.4389 & 0.6276 & 0.7613 & 0.7648 \\
& 0.3 & 0.3867 & 0.8148 & 0.8148 & 1.1229 & 1.3296 & 1.3413 \\
& 0.05 & 0.0101 & 0.0197 & 0.0317 & 0.0354 & 0.0407 & 0.0557 \\
1.5 & 0.1 & 0.0402 & 0.0754 & 0.1198 & 0.1326 & 0.1505 & 0.2015 \\
& 0.2 & 0.1470 & 0.2642 & 0.4052 & 0.4410 & 0.4911 & 0.6315 \\
& 0.3 & 0.2986 & 0.5138 & 0.7601 & 0.8192 & 0.9009 & 1.1292 \\
& 0.05 & 0.0088 & 0.0141 & 0.0231 & 0.0304 & 0.0355 & 0.0355 \\
2.0 & 0.1 & 0.0353 & 0.0550 & 0.0880 & 0.1155 & 0.1328 & 0.1328 \\
& 0.2 & 0.1305 & 0.1971 & 0.3050 & 0.3933 & 0.4417 & 0.4421 \\
& 0.3 & 0.2682 & 0.3920 & 0.5865 & 0.7411 & 0.8197 & 0.8214 \\
\hline
\end{tabular}

Table 2

The first six natural frequency parameters of thick clamped thick plates for different b/a ve t/a ratios

\begin{tabular}{llcccccc}
\hline \multirow{2}{*}{$\mathrm{b} / \mathrm{a}$} & $\mathrm{t} / \mathrm{a}$ & \multicolumn{7}{c}{$\lambda_{i}=\omega \mathrm{t} \sqrt{\frac{\rho}{E}}$} \\
\cline { 3 - 8 } & & $\lambda_{1}$ & $\lambda_{2}$ & $\lambda_{3}$ & $\lambda_{4}$ & $\lambda_{5}$ & $\lambda_{6}$ \\
\hline \multirow{3}{*}{1.0} & 0.05 & 0.0259 & 0.0519 & 0.0519 & 0.0752 & 0.0906 & 0.0912 \\
& 0.1 & 0.0967 & 0.1844 & 0.1844 & 0.2585 & 0.3046 & 0.3075 \\
& 0.2 & 0.3153 & 0.5503 & 0.5503 & 0.7390 & 0.8421 & 0.8538 \\
& 0.3 & 0.5735 & 0.9465 & 0.9465 & 1.2483 & 1.4032 & 1.4239 \\
& 0.05 & 0.0195 & 0.0300 & 0.0470 & 0.0474 & 0.0563 & 0.0708 \\
1.5 & 0.1 & 0.0741 & 0.1114 & 0.1683 & 0.1710 & 0.1992 & 0.2472 \\
& 0.2 & 0.2486 & 0.3604 & 0.5070 & 0.5255 & 0.5920 & 0.7214 \\
& 0.3 & 0.4617 & 0.6525 & 0.8751 & 0.9220 & 1.0195 & 1.2321 \\
& 0.05 & 0.0178 & 0.0230 & 0.0322 & 0.0452 & 0.0455 & 0.0504 \\
2.0 & 0.1 & 0.0678 & 0.0866 & 0.1194 & 0.1635 & 0.1645 & 0.1797 \\
& 0.2 & 0.2287 & 0.2877 & 0.3860 & 0.4933 & 0.5128 & 0.5395 \\
& 0.3 & 0.4256 & 0.5312 & 0.6995 & 0.8513 & 0.9082 & 0.9325 \\
\hline
\end{tabular}

each other as the value of $t / a$ decreases. This shows that the curves of the frequency parameters will almost coincide with each other when the value of the thickness/span ratio, $t / a$, decreases more. In other words, the decrease in the thickness/span ratio will not affect the frequency parameters after a determined value of $t / a$.

\subsubsection{Forced vibration results}

One of the purposes of this paper was to determine the time histories of the displacements and the bending moments at different points of the thick plates subjected to earthquake excitations, but presentation of all of the time histories would take up excessive space. Hence, only the absolute maximum displacements and bending moments for different thickness/span ratio and aspect ratio are presented after two time histories are given. This simplification of presenting only the maximum responses is supported by the fact that the maximum values of these quantities are the most important ones for design. These results are presented in graphical form rather than in tabular form.

The time histories of the center displacements of the thick simply supported plates for $b / a=1.0$, and 2.0 when $t / a=0.2$ are given in Fig. 6(a) and 6(b), respectively. As seen from Fig. 6(a) and 6(b), the center displacements of the thick simply supported plates for $b / a=1$, and $t / a=0.2$, and for $b / a=2$, and $t / a=0.2$, reached their absolute maximum values of $0.00631 \mathrm{~mm}$ at $3.47 \mathrm{~s}$, and of $0.01447 \mathrm{~mm}$ at $3.48 \mathrm{~s}$, respectively. These absolute maximum values are different even with the same occurring time as the dynamic characteristics of the thick plates affect the response. It is also understandable that the system becomes more flexible as the aspect ratio increases.

The absolute maximum displacements of the thick simply supported plates for different aspect ratios, and thickness/span ratios are given in Fig. 7. As seen from Fig. 7, the absolute maximum displacements of the thick simply supported plates increase with increasing aspect ratio for a constant t/a ratio. The same displacements decrease with 

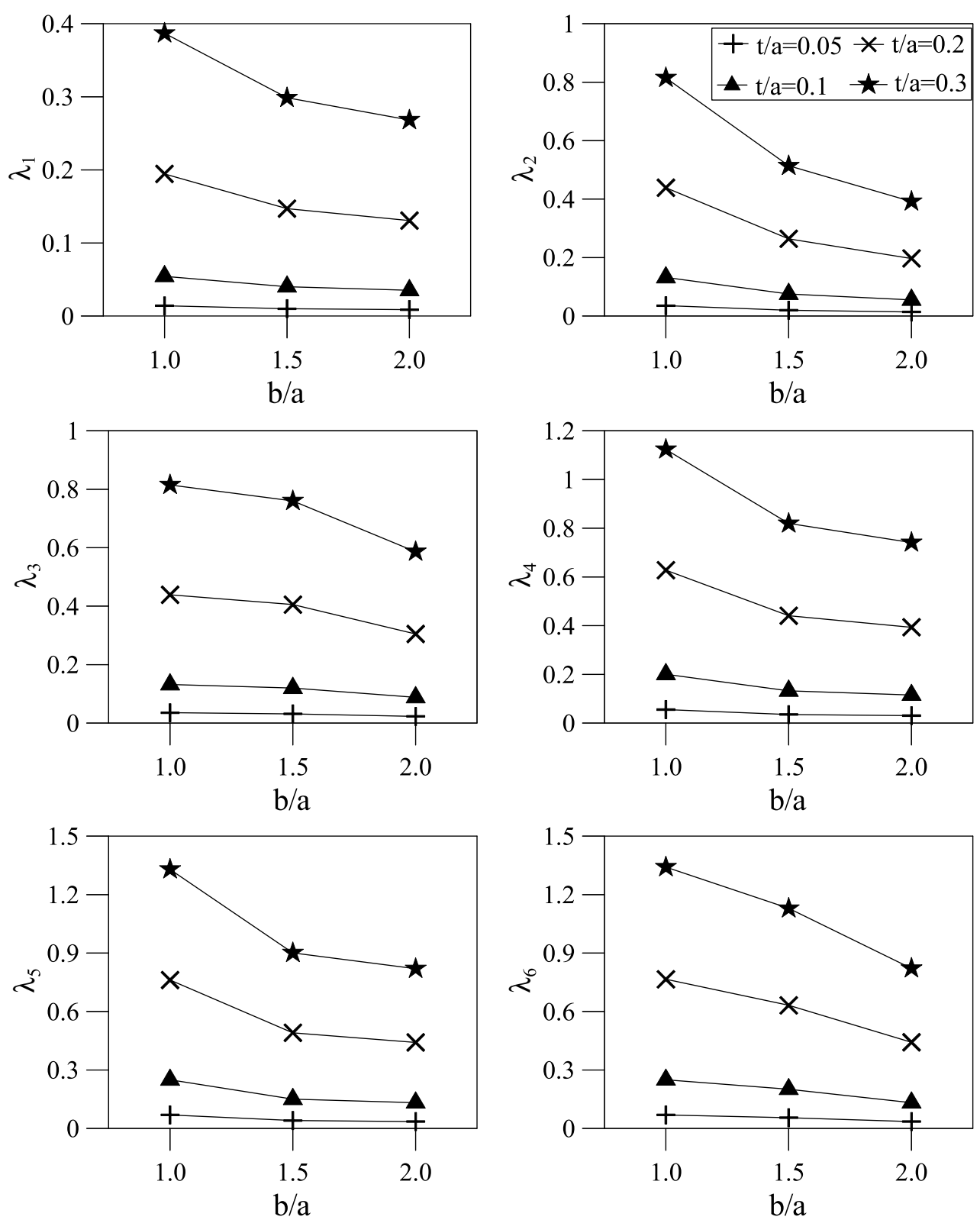

Fig. 4. Effects of aspect ratio and thickness/span ratio on the first six frequency parameters of the thick simply supported plates.

increasing t/a ratio for a constant b/a ratio. As also seen from this figure, the decrease in the absolute maximum displacement for a constant $b / a$ ratio increases with increasing b/a ratio. The curves for a constant value of the aspect ratio, $b / a$ are fairly getting closer to each other as the value of $t / a$ increases. This shows that the curves of the absolute maximum displacements will almost coincide with each other when the value of the thickness/span ratio, $t / a$, increases more. In other words, the increase in the thickness/span ratio will not affect the absolute maximum displacements after a determined value of $t / a$. In general, the effects of the changes in the thickness/span ratios on the absolute maximum displacement are larger than the changes in the aspect ratios.

The absolute maximum bending moments $M_{x}$ at the center of the thick simply supported plates for different aspect ratios and thickness/span ratios are given in Fig. 8. As seen from Fig. 8, the absolute maximum bending moment, $M_{x}$, at the center of the thick simply supported plates increases with increasing aspect ratio and thickness/span ratio. 

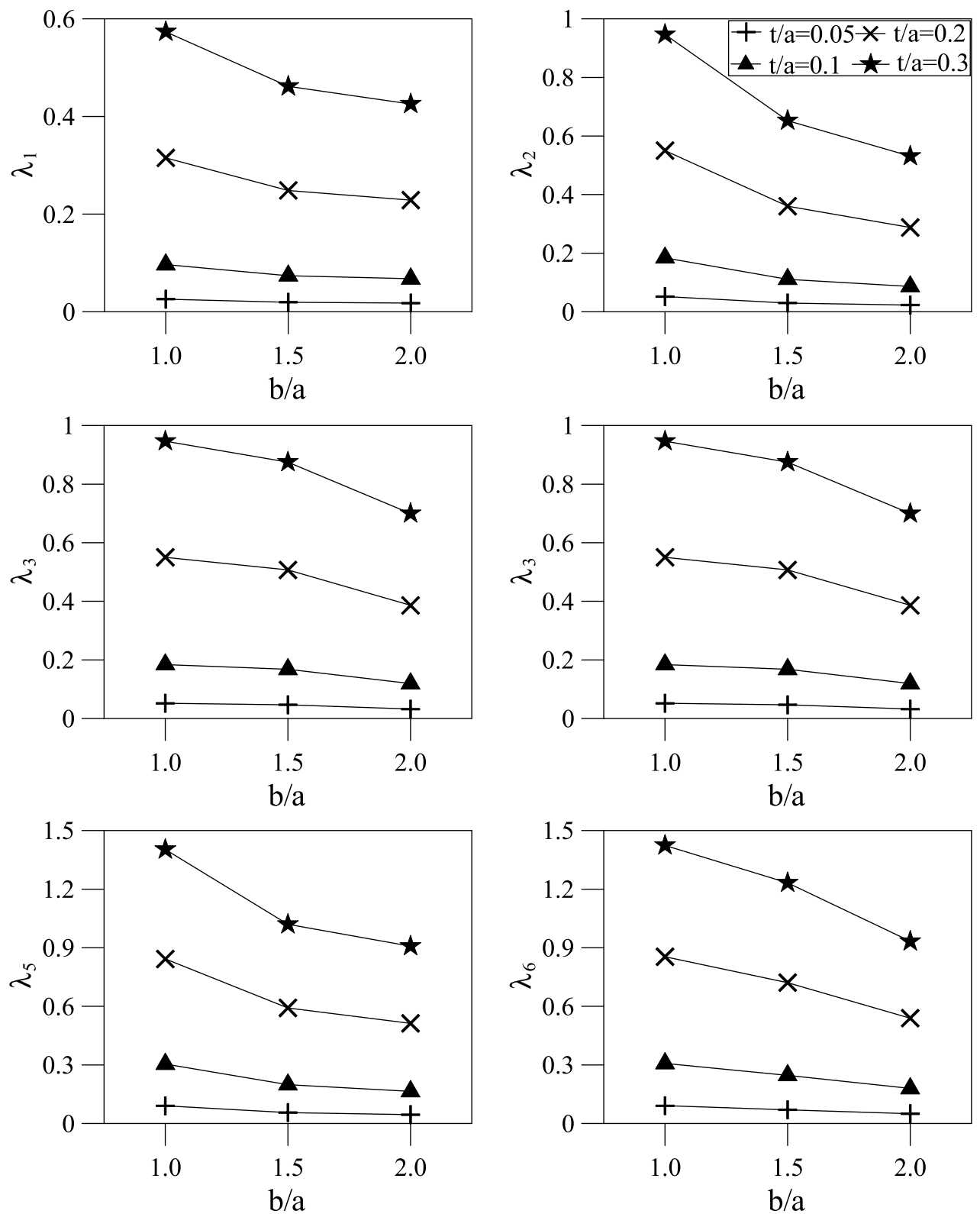

Fig. 5. Effects of aspect ratio and thickness/span ratio on the first six frequency parameters of the thick clamped plates.

The increases in the absolute maximum bending moment, $M_{x}$, increase with increasing aspect and thickness/span ratios. This is understandable that increasing the aspect ratio makes the plate stiffer in the short span, the $\mathrm{x}$ axis, direction. As also seen from this figure, in general, the effects of the changes in the aspect ratios on the absolute maximum bending moment, $\mathbf{M}_{x}$, are larger than the changes in the thickness/span ratios.

The absolute maximum bending moments $M_{y}$ at the center of the thick simply supported plates for different aspect ratios and thickness/span ratios are given in Fig. 9. As seen from Fig. 9, the absolute maximum bending moment, $M_{y}$, at the center of the thick simply supported plates decreases with increasing aspect ratio and increases with increasing thickness/span ratio. The decrease in the absolute maximum bending moment, $\mathbf{M}_{y}$, increase with increasing aspect ratio. The increase in the absolute maximum bending moment, $M_{y}$, increases with increasing thickness/span ratios. This is understandable that increasing the aspect ratio makes the thick plate more flexible in 


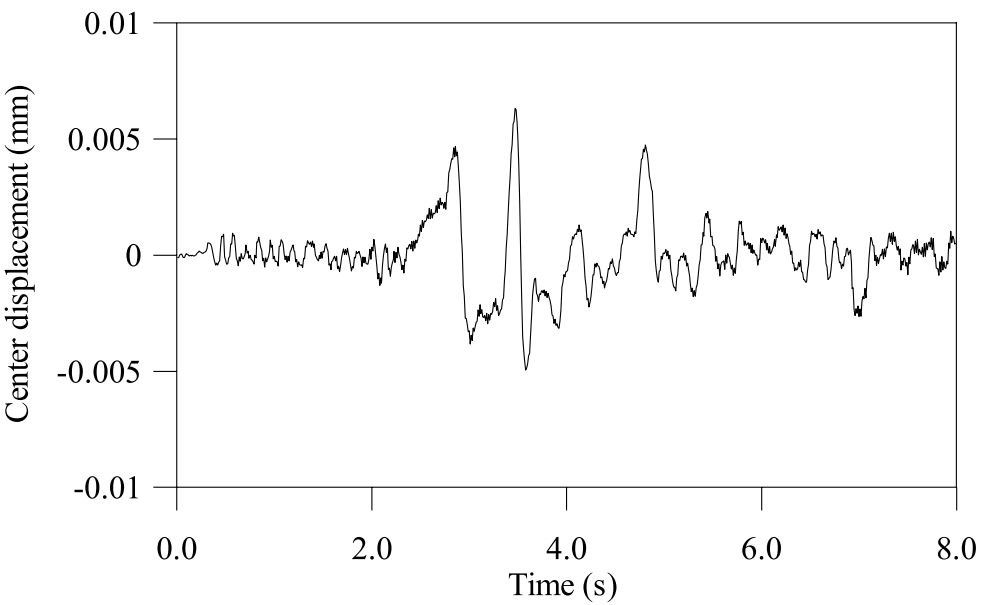

(a)

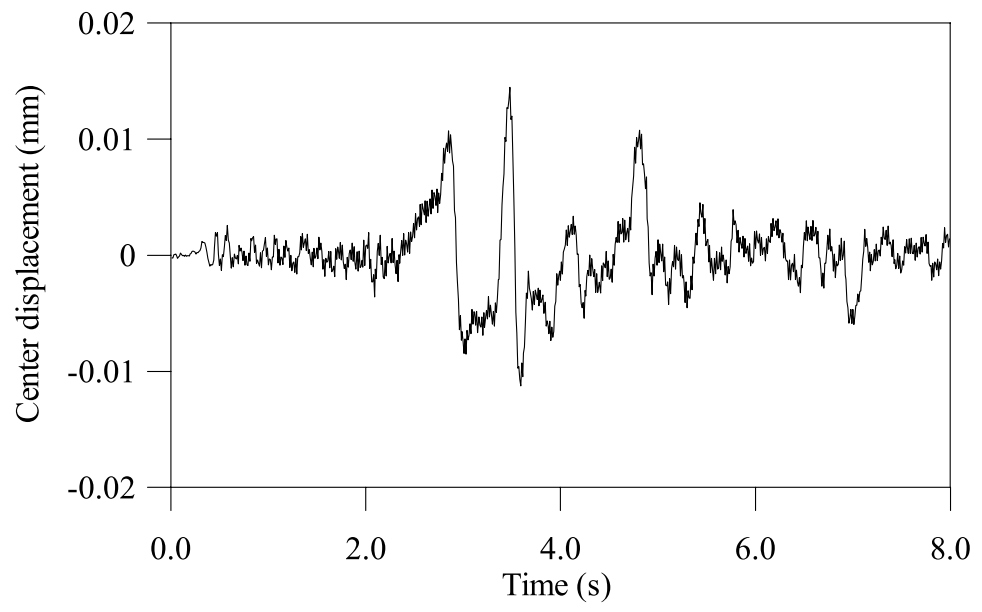

(b)

Fig. 6. The time history of the center displacement of the thick clamped plate for (a) $b / a=1.0$ and $t / a=0.2$, and (b) $b / a=2.0$ and $t / a=0.2$.

the long span, the $y$ axis, direction. As also seen from this figure, in general, the effects of

the changes in the thickness/span ratios on the absolute maximum bending moment, $M_{y}$, are larger than the changes in the aspect ratios.

It should be noted that, in this study, the thick plates clamped along all four edges are also analyzed. Since the variations in the responses of the thick clamped plates with the thickness/span ratios, and the aspect ratios are similar to the variations in the responses of the thick simply supported plates with the same parameters, the results of the thick clamped plates are not presented. It should also be noted that the absolute maximum displacements of the thick simply supported plates are larger than those of the thick clamped plates for the same aspect and thickness/span ratios. Since no results have been found in the literature for the case of the same earthquake problem, comparison of the results obtained in this study is not possible.

\section{Conclusions}

For a thick plate subjected to the earthquake excitations, it is somewhat difficult to interpret the effects of the thickness/span ratio, the aspect ratio, and the boundary conditions on the responses because both the frequency content of the earthquake excitation and the exact natural frequency of the particular thick plate can make a difference 


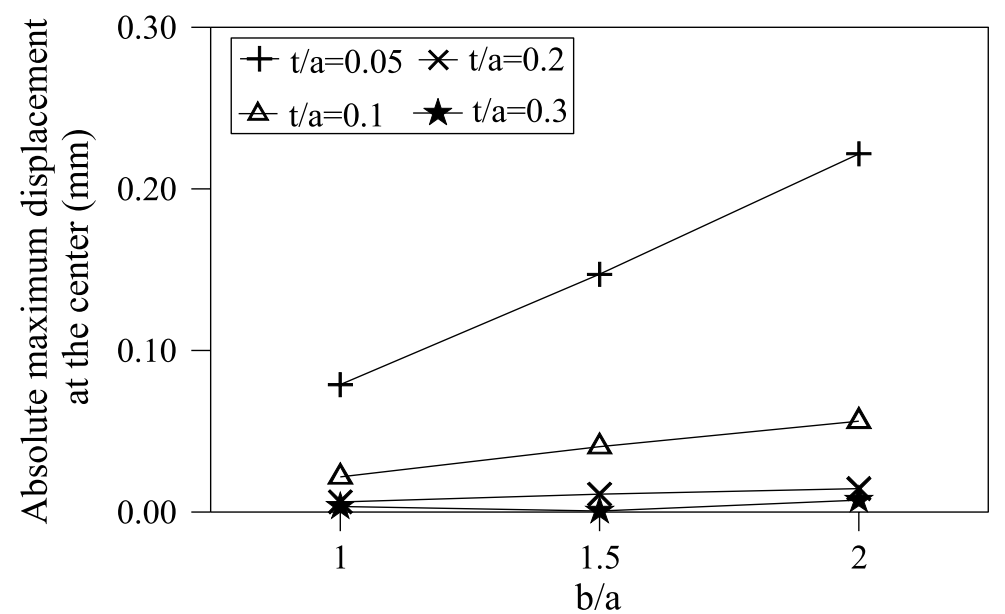

Fig. 7. Absolute maximum displacement of the thick simply supported plates for different aspect ratios and thickness/span ratios.

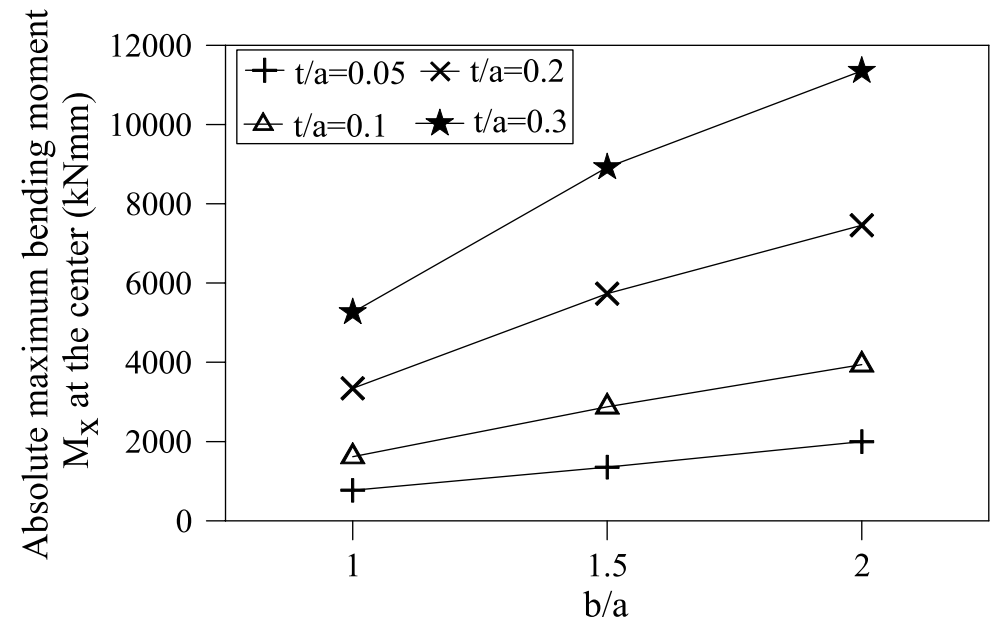

Fig. 8. Absolute maximum bending moment $M_{x}$ at the center of the thick simply supported plates for different aspect ratios and thickness/span ratios.

to its response. In order to generalize the results obtained in this study, the responses of the different thick plates subjected to different earthquake excitations should be evaluated all together. Therefore, the curves presented herein can help the designer to anticipate the effects of the thickness/span ratio, the aspect ratio, and the boundary conditions on the earthquake response of a thick plate. The coded program can be effectively used for the free vibration and earthquake analyses of any thick plates. In addition, the following conclusions can also be drawn from the results obtained in this study.

The increase in the frequency parameters with increasing $t / a$ ratio for a constant value of $b / a$ ratio gets larger for larger values of the frequency parameters. The effects of the change in the $t / a$ ratio on the frequency parameter of the thick plates are always larger than those of the change in the aspect ratio. The absolute maximum displacements of the thick plates increase with increasing aspect ratio for a constant t/a ratio. The same displacements decrease with increasing t/a ratio for a constant b/a ratio. The effects of the changes in the thickness/span ratios on the absolute maximum displacement are generally larger than the changes in the aspect ratios. The absolute maximum bending moment, $M_{x}$, at the center of the thick plates increases with increasing aspect ratio and thickness/span ratio. The effects of the changes in the aspect ratios on this $M_{x}$ are generally larger than the changes in the thickness/span ratios. The absolute maximum bending moment, $M_{y}$, at the center of the thick plates decreases with increasing 


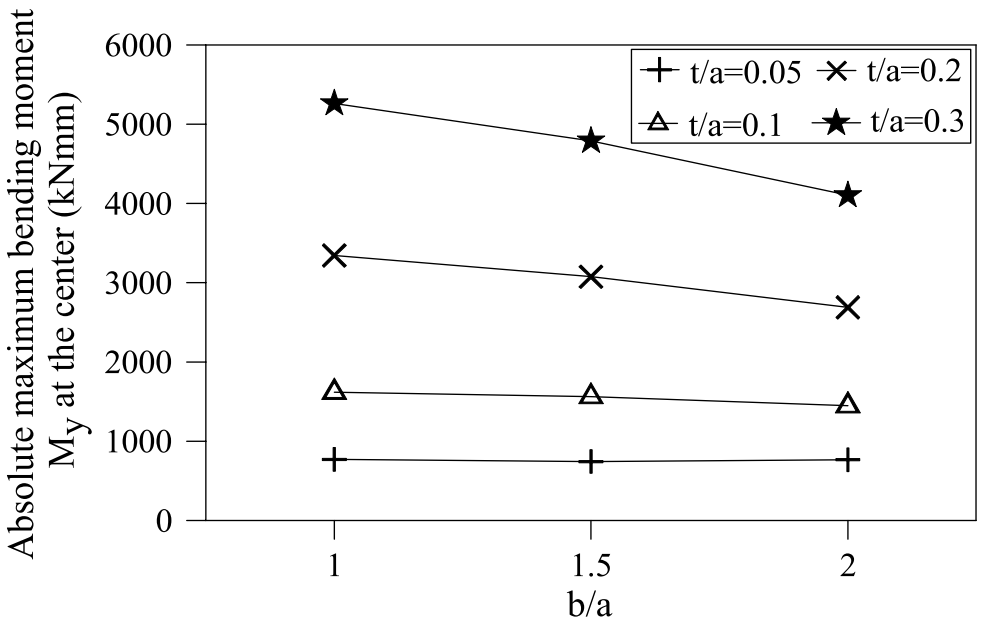

Fig. 9. Absolute maximum bending moment $M_{y}$ at the center of the thick simply supported plates for different aspect ratios and thickness/span ratios.

aspect ratio and increases with increasing thickness/span ratio. The effects of the changes in the thickness/span ratios on this $\mathrm{M}_{y}$ are generally larger than the changes in the aspect ratios. In general, degrees of decreases and increases depend on the changes in the aspect and thickness/span ratios, and the changes in the thickness/span ratio are more effective on the maximum responses considered in this study than the changes in the aspect ratio.

\section{Acknowledgements}

This study is a part of Ph. D. Thesis of Yaprak Itır Özdemir, and it is supported by the Research Fund of Karadeniz Technical University. Project number: 2002.112.1.5.

\section{References}

[1] A.C. Ugural, Stresses in Plates and Shells, McGraw-Hill., New York, 1981.

[2] A.K. Soh, S. Cen, Y. Long and Z. Long, A new twelve DOF quadrilateral element for analysis of thick and thin plates, European Journal of Mechanics; A/Solids 20 (2001), 299-326.

[3] A.W. Leissa, Vibration of plates, NASA, sp. 160, 1969.

[4] A.W. Leissa, The free vibration of rectangular plates, Journal Sound and Vibration 31(3) (1973), $257-294$.

[5] C. Wanji and Y.K. Cheung, Refined quadrilateral element based on Mindlin/Reissner plate theory, International Journal for Numerical Methods in Engineering 47 (2000), 605-627.

[6] C.P. Providakis and D.E. Beskos, Free and forced vibrations of plates by boundary elements, Computer Methods in Applied Mechanics and Engineering 74 (1989), 231-250.

[7] C.P. Providakis and D.E. Beskos, Free and forced vibrations of plates by boundary and interior elements, International Journal for Numerical Methods in Engineering 28 (1989), 1977-1994.

[8] E. Hinton and H.C. Huang, A Family of Quadrilateral Mindlin Plate Element with Substitute Shear Strain Fields, Computer and Structures 23(3) (1986), 409-431.

[9] E. Reissner, The Effect of Transverse Shear Deformation on the Bending of Elastic Plates, Journal of Applied Mechanics (ASME) 12 (1945), A69-A77.

[10] E. Reissner, On Bending of Elastic Plates, Quarterly of Applied Mathematics 5(1) (1947), 55-68.

[11] E. Reissner, On a Variational Theorem in Elasticity, Journal of Mathematics and Physics 29 (1950), 90-95.

[12] F. Brezzi and L.D. Marini, A nonconforming element for the Reissner-Mindlin plate, Computer and Structure 81 (2003), 515-522.

[13] G.B. Warburton, The vibration of rectangular plates, Proceeding of the Institute of Mechanical Engineers 168 (1954), $371-384$.

[14] G.W. Caldersmith, Vibrations of orthotropic rectangular plates, ACUSTICA 56 (1984), 144-152.

[15] H.S. Shen, J. Yang and L. Zhang, Free and forced vibration of Reissner-Mindlin plates with free edges resting on elastic foundation, Journal of Sound and Vibration 244(2) (2001), 299-320.

[16] J. Qiu and Z.C. Feng, Parameter dependence of the impact dynamics of thin plates, Computer and Structures 75(5) (2000), 491-506. 
[17] J. Zhu and P. Gu, Dynamic response of orthotropic plate using BEM with approximate fundamental solution, Journal of Sound and Vibration 151(2) (1991), 203-211.

[18] K.J. Bathe, Finite Element Procedures, Prentice Hall, Upper Saddle River, New Jersey, 1996

[19] K.K. Raju and E. Hinton, Natural Frequencies and Modes of Rhombic Mindlin Plates, Earthquake Engineering and Structural Dynamics 8 (1980), 55-62

[20] K.M. Liew and T.M. Teo, Three-dimensional vibration analysis of rectangular plates based on differential quadrature method, Journal of Sound and Vibration 220(4) (1999), 577-599.

[21] K.S. Woo, C.H. Hong, P.K. Basu and C.G. Seo, Free vibration of skew Mindlin plates by p-version of F.E.M., Journal of Sound and Vibration 268 (2003), 637-656.

[22] L. Belounar and M. Guenfound, A new rectangular finite element based on the strain approach for plate bending, Thin-Walled Structures 43 (2005), 47-63.

[23] L. Cai, T. Ron and D. Chen, Generalized Mixed Variational Methods for Reissner Plate and Its Application, Computational Mechanics 30 (2002), 29-37.

[24] L.F. Qian, R.C. Batra and L.M. Chen, Free and forced vibration of thick rectangular plates using higher-order shear and normal deformable plate theory and meshless Petrov-Galerkin (MLPG) method, Computer Modeling in Engineering \& Sciences 4(5) (2003), 519-534.

[25] M.V.G. Morais, L.J. Pedroso and S.F. Da Silva, Passager de Paris 1 (2005), 238-250.

[26] O.C. Zienkiewich, R.L. Taylor and J.M. Too, Reduced integration technique in general analysis of plates and shells, International Journal for Numerical Methods in Engineering 3 (1971), 275-290.

[27] P.G. Bergan and X. Wang, Quadrilateral Plate Bending Elements with Shear Deformations, Computer and Structures 19(1-2) (1984), 25-34.

[28] R.D. Cook, D.S. Malkus and E.P. Michael, Concepts and Applications of Finite Element Analysis, John Wiley \& Sons, Inc., Canada, 1989.

[29] R.D. Mindlin, Influence of Rotatory Inertia and Shear on Flexural Motions of Isotropic, Elastic Plates, Journal of Applied Mechanics 18(1) (1951), 31-38.

[30] R.M. Grice and R.J. Pinnington, Analysis of the flexural vibration of a thin-plate box using a combination of finite element analysis and analytical impedances, Journal of Sound and Vibration 249(3) (2002), 499-527.

[31] S. Cen, Y.Q. Long, Z.H. Yao and S.P. Chiew, Application of the quadrilateral area co-ordinate method: Anew element for Mindlin-Reissner plate, International Journal for Numerical Methods in Engineering 66 (2006), 1-45.

[32] S. Timoshenko and S. Woinowsky-Krieger, Theory of Plates and Shells, Second edition, McGraw-Hill., New York, 1959.

[33] T. Sakata and K. Hosokawa, Vibrations of clamped orthotropic rectangular plates, Journal of Sound and Vibration 125(3) (1988), 429-439.

[34] T.A. Ozkul and U. Ture, The transition from thin plates to moderately thick plates by using finite element analysis and the shear locking problem, Thin-Walled Structures 42 (2004), 1405-1430.

[35] T.J.R. Hughes, R.L. Taylor and W. Kalcjai, Simple and efficient element for plate bending, International Journal for Numerical Methods in Engineering 11 (1977), 1529-1543.

[36] T.S. Lok and Q.H. Cheng, Free and forced vibration of simply supported, orthotropic sandwich panel, Computer and Structures 79(3) (2001), 301-312.

[37] W. Weaver and P.R. Johnston, Finite Elements for Structural Analysis, Prentice Hall, Inc., Englewood Cliffs, New Jersey, 1984.

[38] W.J. Si, K.Y. Lam and S.W. Gang, Vibration analysis of rectangular plates with one or more guided edges via bicubic B-spline method, Shock and Vibration 12(5) (2005).

[39] Y. Ayvaz, Parametric Analysis of Reinforced Concrete Slabs Subjected to Earthquake Excitation, Ph. D. Dissertation, Texas Tech University, 1992.

[40] Y. Ayvaz, A. Daloğlu and A. Doğangün, Application of a Modified Vlasov Model to Earthquake Analysis of the Plates Resting on Elastic Foundations, Journal of Sound and Vibration 212(3) (1998), 499-509.

[41] Y.I. Özdemir and Y. Ayvaz, Shear Locking-Free Analysis of Thick Plates Using Mindlin's Theory, Structural Engineering and Mechanics 27(3) (2007), 311-331.

[42] Y.I. Özdemir, Parametric Analysis of Thick Plates Subjected to Earthquake Excitations by Using Mindlin's Theory, Ph. D. Dissertation, Karadeniz Technical University, 2007. 

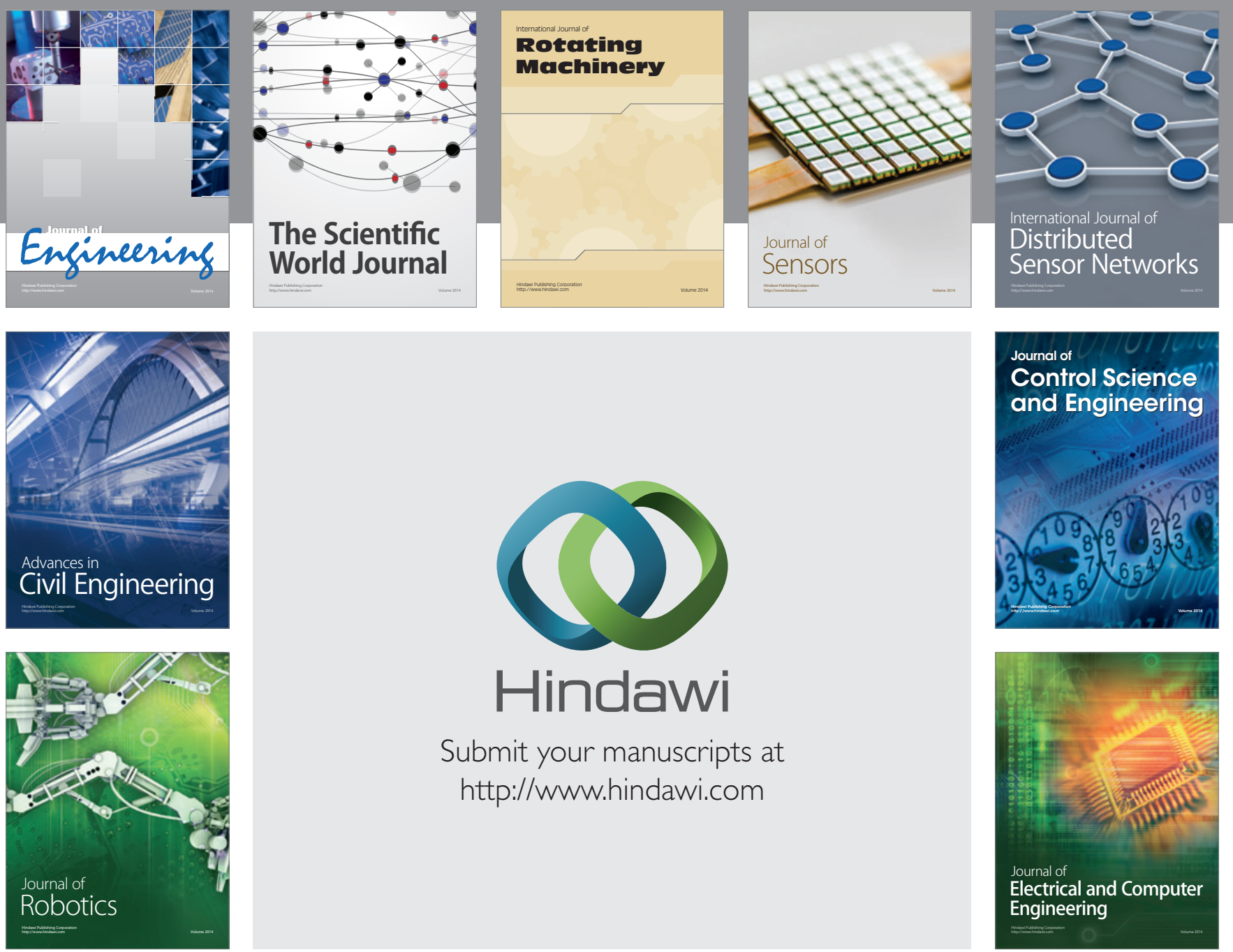

Submit your manuscripts at

http://www.hindawi.com
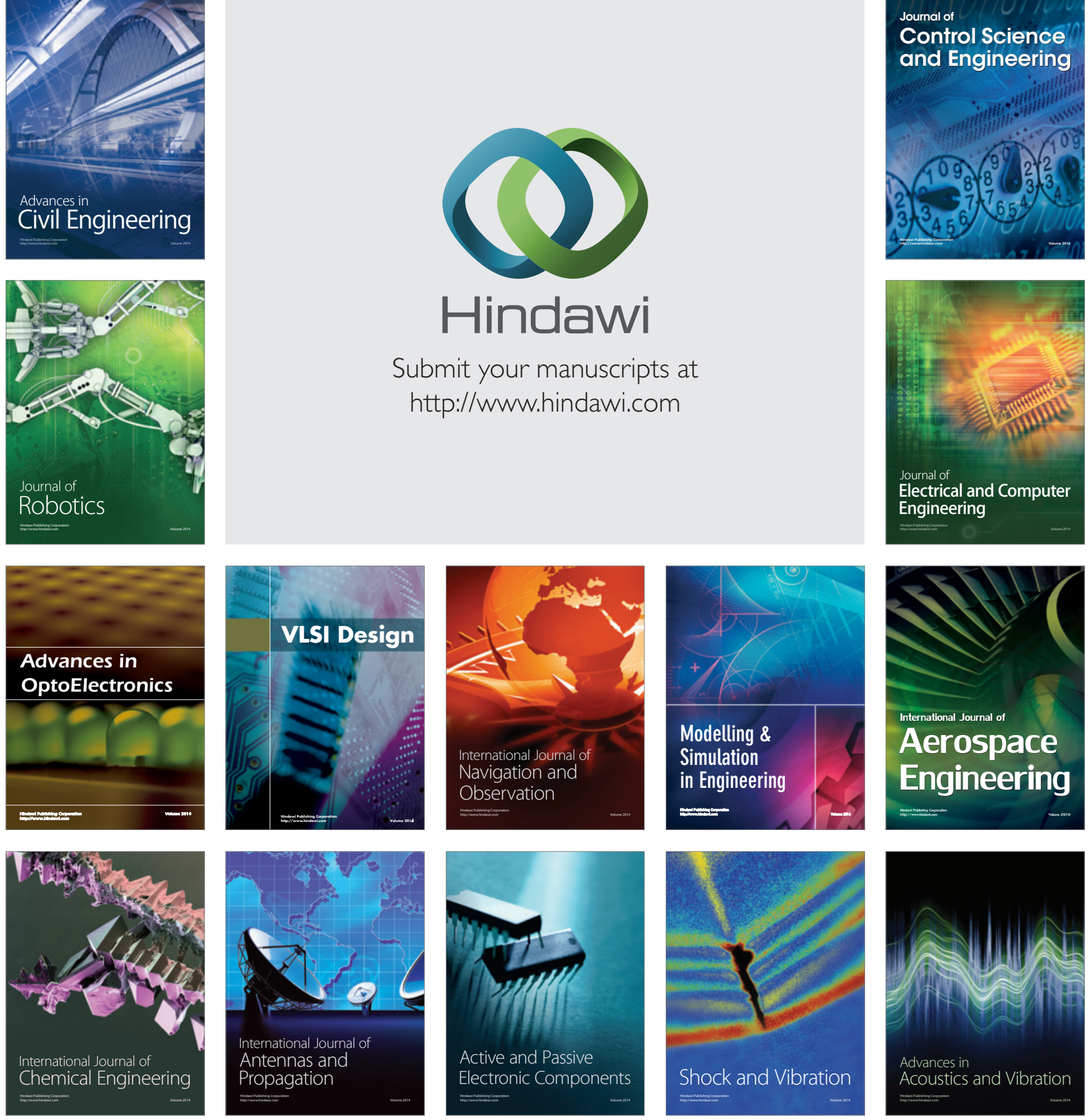